\title{
Chronic hypotension and modulation of autonomic cardiovascular regulation
}

\author{
Gianfranco Parati, Marco Di Rienzo, Paolo Coruzzi and Paolo Castiglioni \\ Hypertension Research (2009) 32, 931-933; doi:10.1038/hr.2009.150; published online 2 October 2009
}

\section{THE PROBLEM OF CHRONIC HYPOTENSION}

Yypotension is defined by WHO 1 (World Health Organization) ${ }^{1}$ as a systolic blood pressure (SBP) lower than $100 \mathrm{~mm} \mathrm{Hg}$ in women and $110 \mathrm{~mm} \mathrm{Hg}$ in men. Chronic hypotension affects quality of life, because of symptoms such as fatigue, dizziness, headache, cold limbs and reduced cognitive performance. ${ }^{2-4}$ Chronic 'essential' hypotension should be distinguished from secondary hypotension (due to, for example, blood loss or the effect of medication) and from orthostatic hypotension, in which blood pressure (BP) decreases only when assuming the upright position. ${ }^{5}$ Understanding the pathophysiology and proper treatment of chronic hypotension benefits affected patients and offers a deeper insight into the physiology of BP control. Alterations in autonomic cardiovascular regulation have been hypothesized to contribute to the pathogenesis of this condition. ${ }^{4,6}$ Such alterations include, in particular, an increase in arterial baroreflex sensitivity (BRS) with arterial baroreflex resetting toward the lower BP values. ${ }^{7,8}$ This pattern is accompanied by reduced sympathetic activity and by enhanced parasympathetic activity. ${ }^{4,9}$

Aimed at opposing these autonomic changes, sympathomimetic drugs are often employed in the treatment of hypotensive disorders, in particular when orthostatic hypotension occurs. Among them, the

$\overline{\text { G Parati is at the Department of Clinical Medicine and }}$ Prevention, University of Milano-Bicocca and at the Department of Cardiology, St Luca Hospital, Istituto Auxologico Italiano, IRCCS, Milan, Italy; M Di Rienzo and P Castiglioni are at the Polo Tecnologico, IRCCS 'Santa Maria Nascente', Fondazione Don C Gnocchi ONLUS, Milan, Italy; P Coruzzi is at the Department of Clinical Sciences, University of Parma and at the Fondazione Don C Gnocchi ONLUS, Parma, Italy.

E-mail: gianfranco.parati@unimib.it $\alpha$-adrenergic agent, midodrine, is frequently prescribed for patients with orthostatic symptoms. ${ }^{10,11}$ Data suggest that midodrine may also have favorable effects in patients with chronically low BP, as it is reported to increase BP, vascular resistance, stroke volume $^{12}$ and cerebral blood flow, which is associated with improved mental performance. ${ }^{13}$ However, limited information is available about the possible effects of drugs that stimulate $\alpha$-adrenergic receptors on autonomic cardiovascular regulation.

This issue of Hypertension Research includes a paper by Duschek et al. ${ }^{14}$ that addresses this topic. The authors investigated the acute effects of a single administration of the $\alpha$-mimetic drug, midodrine, on baroreflex function, as well as on sympathetic and parasympathetic cardiac control in subjects with chronic hypotension. Spontaneous BRS was assessed by the sequence technique, ${ }^{15,16}$ and additional information on cardiac autonomic modulation was obtained through spectral analysis of heart rate variability (HRV), by focusing on high-frequency (HF) and low-frequency (LF) components and on the $\mathrm{LF} / \mathrm{HF}$ ratio. ${ }^{17}$ Data were obtained at rest and under mental stress, through application of a mental arithmetic task, in 50 patients with chronic hypotension (mean SBP/diastolic $\mathrm{BP}(\mathrm{DBP})=96 / 61 \mathrm{~mm} \mathrm{Hg})$, according to a placebo-controlled double-blind study. Acute midodrine treatment did increase BP, but it also increased BRS and parasympathetic cardiac modulation (as indirectly indicated by an increase in HF and a decrease in the LF/ HF power ratio). The interpretation given by Duschek et al. ${ }^{14}$ is that the BP increase induced by acute midodrine administration elicits a counter-regulatory autonomic response aimed at returning BP to the initial value, which involves a shift in the autonomic balance toward less sympathetic and more parasympathetic activity. Owing to these accompanying changes, Duschek et al. conclude that their data challenge the use of $\alpha$-sympathomimetics in the treatment of chronic hypotension, given that their administration may not reduce but rather worsen the autonomic dysregulation associated with this condition.

\section{CHRONIC HYPOTENSION TREATMENT AND AUTONOMIC CARDIOVASCULAR REGULATION}

Proper interpretation of the findings by Duschek et al. ${ }^{14}$ requires adequate information on the patterns that characterize changes in autonomic drive after administration of treatment for chronic hypotension. This might allow identification of drugs that improve the hemodynamic condition and preserve a balanced autonomic profile.

Several methods are available to investigate autonomic cardiovascular regulation in humans. Precise information may be obtained through direct recording of sympathetic outflow with microneurographic methods. However, microneurography provides a measure of the sympathetic drive only to certain muscular districts, which may not reflect actual sympathetic regulation of the heart and vessels. Moreover, these recordings are technically difficult to obtain and require skilled operators. In practice, such recordings cannot be easily employed over long time periods for monitoring the effects of antihypotensive drugs on autonomic cardiovascular regulation. Alternatively, the analysis of spontaneous HR and BP variability offers valuable information on cardiac parasympathetic, cardiac sympathetic and vascular sympathetic regulation. ${ }^{17}$ Beat-to-beat time series of BP and HR can be easily and noninvasively recorded in most clinical settings. However, as BP and HR variability provide 
only indirect information on autonomic cardiovascular control, and given the strong interaction between the autonomic nervous system and other physiological systems, great methodological care is needed to correctly interpret the results. Among the indexes of HRV, the clearest information on autonomic cardiac modulation is provided by the HR power in the HF region. The HF power is linked to the strength of cardiac vagal drive ${ }^{17}$ and mostly reflects the HR fluctuations associated with respiration. Central oscillators may contribute to the respiratory sinus arrhythmia, but certainly a major role is played by the arterial baroreflex, which triggers changes in cardiac interval following BP oscillations produced by respiratory changes in intrathoracic pressure. ${ }^{18}$ This reflex mechanism may largely prevail over central components, at least in certain animal species. ${ }^{19}$ If the breathing frequency is higher than $0.15 \mathrm{~Hz}$, the respiratory sinus arrhythmia is entirely modulated by the vagal outflow, because the sympathetic drive is too sluggish to modulate fast fluctuations ${ }^{17}$ and the HF power largely reflects the vagal drive. This is the approach followed by Duschek et al. ${ }^{14}$ to quantify changes in cardiac vagal modulation after acute midodrine administration. When focussing on HF heart rate powers, however, it is important to monitor the actual breathing rate, particularly when subjects are allowed to breathe spontaneously. In general, spontaneous breathing seems to be preferable when assessing HRV parameters, because breathing at a paced controlled rate (for instance, following a metronome) may be responsible for some degree of autonomic activation (due to mental stress or chemoreceptor stimulation), which may itself affect autonomic cardiovascular control. However, when subjects breathe freely, the respiration frequency should be measured to verify that respiration actually falls in the HF region. This measure can also exclude the possibility that pharmacological treatment itself-alone or in combination with other factors-might induce changes in breathing rate. In the case of pharmacological treatment, small variations in $\mathrm{HF}$ power of $\mathrm{HR}$ might not reflect changes in cardiac vagal drive. Depending on the breathing rate observed before and during treatment, changes in HF power of HR may simply result from differences in the gain of the mechanical transfer function between intrathoracic pressure and arterial pressure, and/or differences in the gain of the baroreflex transfer function between arterial pressure and HR. ${ }^{20}$ Although breathing rate was not measured in this study, the substantial increase in HF power and the parallel increase in mean $\mathrm{R}-\mathrm{R}$ interval observed by Duschek et al. ${ }^{14}$ are convincing evidence of an increased cardiac vagal drive after midrodine administration.

Other parameters of primary importance are the BP and HR spectral powers in the LF region, a relatively narrow band centered around $0.1 \mathrm{~Hz}$. The power in this band should quantify the intensity of BP (and consequently HR) modulations in the frame of a physiological phenomenon called 'ten-second rhythm,' or 'Mayer waves' (for BP). The phenomenon is defined by waxing and waning fluctuations in BP and HR, which do not have a deterministic nature, as in the case of respiratory oscillations. Rather, they are better described as a stochastic, narrowband colored noise, with maximal power at $0.1 \mathrm{~Hz}$. Compared with respiratory oscillations, the origin of Mayer waves in $\mathrm{BP}$ is more controversial. ${ }^{21}$ It has been hypothesized that they are generated by central oscillators, but strong evidence also suggests that they are produced by a resonance of the baroreflex loop at $0.1 \mathrm{~Hz}$. Whatever the origin-central or reflex-of the Mayer waves might be, the $0.1 \mathrm{~Hz}$ oscillations in HR seem to be mainly due to baroreflex modulation: baroreceptors sense the Mayer BP waves and modulate the firing rate of the sinus node synchronously. This origin is supported by the observation that in spinal-cord-injured subjects with low-level lesion, the ten-second rhythm disappears not only in DBP (as expected because of the impaired vascular sympathetic modulation) but also in HR, although the cardiac sympathetic efferent fibers are intact. ${ }^{22}$ As the baroreceptors modulate $\mathrm{HR}$ at $0.1 \mathrm{~Hz}$ by decelerating vagal outflow and accelerating sympathetic outflow, the HR LF power does not represent a specific index of cardiac sympathetic drive. Aimed at obtaining more reliable information on the balance between sympathetic and parasympathetic cardiac modulation, certain studies recommend calculation of the ratio between LF and HF HRV powers. ${ }^{17}$ Duschek et al. ${ }^{14}$ report a significant decrease in the LF/HF HRV power ratio after midodrine treatment, which they interpret as a shift of the cardiac sympathovagal balance toward greater vagal modulation. However, a yet unanswered question is whether the change in LF/HF power ratio is due to an increased vagal drive only, or to a decreased cardiac sympathetic drive without changes in the vascular sympathetic outflow, or, alternatively, to an overall decrease of sympathetic (both cardiac and vascular) modulation. Assessment of LF power of DBP, the indirect index of vascular sympathetic modulation of peripheral resistance, might help to clarify this issue in future studies.

The simultaneous analysis of BP and HR variability also allows us to estimate the sensitivity of baroreflex control of $\mathrm{HR}$ (BRS). Several methods have been proposed to this end, ${ }^{23,24}$ each method differing in terms of its applicability (requirement of a stationary signal, time resolution of the estimate, number of estimates per hour, reliability, reproducibility, emphasis on vagal or sympathetic control, applicability in elderly subjects or dysautonomic patients, etc.).

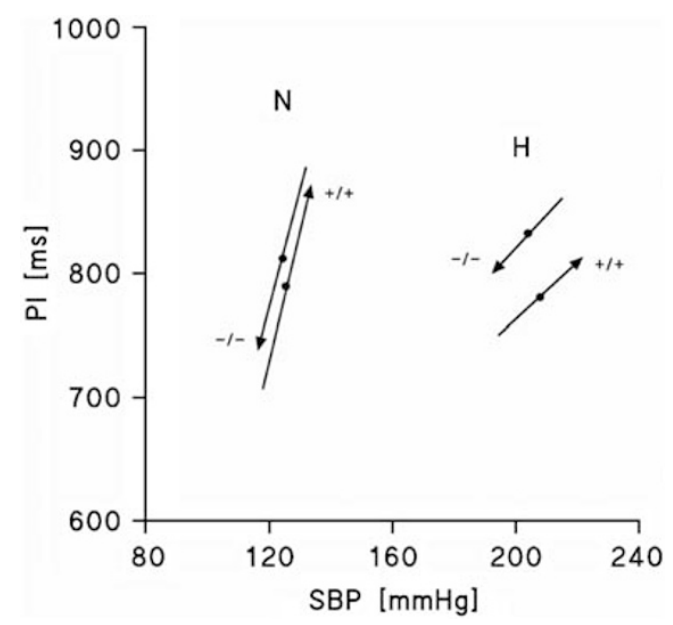

Figure 1 Sequence analysis on 4-h arterial blood pressure recordings during nighttime (2300-0300 h) in a normotensive $(\mathrm{N})$ subject and a severely hypertensive $(\mathrm{H})$ subject. Arrows show the average of $+/+$ sequences (sequences in which a SBP increase is followed by an increase in pulse interval, $\mathrm{PI}$ ) and $-/$ - sequences (in which an SBP decrease is followed by PI shortening). Please note that sequence analysis may provide information not only on BRS (slope of the lines) but also on working point (solid circles) and amplitude of the input/output relationship (Parati G, Castiglioni P, Di Rienzo M, data on file). 
Thus, researchers have a number of tools available. Duschek et al. ${ }^{14}$ applied one of the more popular techniques, the sequence method, which calculates BRS as the slope of the regression line between SBP and cardiac interval values in sequences of beats characterized by simultaneous increases or decreases in SBP and R-R interval. ${ }^{25}$ They found that the high BRS values of chronic hypotension subjects increased even more with midodrine. This increase supports the presence of increased vagal cardiac drive and can be logically explained as an acute counter-measure against the BP increase induced by the $\alpha$-adrenergic treatment. Still, such a large increase is surprising, because one might expect that hours after midodrine administration, the baroreflex working point would shift close to the saturation point on the baroreceptor input/output sigmoidal curve. This shift is even more likely to occur in the case of chronic treatment, a possibility that was not verified in this study.

Therefore, the interesting results published by Duschek et al. certainly deserve to be further investigated in future studies. This can be done, on one hand, by exploiting other features of the baroreflex function. For instance, the same sequence technique also provides estimates of the baroreflex effectiveness index (ratio between generated sequences and SBP input ramps), ${ }^{25}$ the response latency, ${ }^{26}$ the SBP excursions around the baroreflex 'set point' (Figure 1) and the characteristics of 'central' sequences. On the other hand, this can be done by considering the effects of long-term drug administration, a common occurrence in clinical practice.

\section{CONFLICT OF INTEREST}

The authors declare no conflict of interest.

1 WHO. Arterial Hypertension, Technical Report series no. 628. World Health Organisation: Geneva, 1978.

2 Rosengren A, Tibblin G, Wilhelmsen L. Low systolic blood pressure and self perceived wellbeing in middle aged men. BMJ 1993; 306: 243-246.

3 Wessely S, Nickson J, Cox B. Symptoms of low blood pressure: a population study. BMJ 1990; 301: 362-365.

4 Duschek S, Schandry R. Reduced brain perfusion and cognitive performance due to constitutional hypotension. Clin Auton Res 2007; 17: 69-76.

5 Low PA, Singer W. Management of neurogenic orthostatic hypotension: an update. Lancet Neurol 2008; 7: 451-458.

6 Weisz N, Schandry R, Jacobs AM, Mialet JP, Duschek S. Early contingent negative variation of the EEG and attentional flexibility are reduced in hypotension. Int $\mathrm{J}$ Psychophysiol 2002; 45: 253-260.

7 Duschek S, Dietel A, Schandry R, Reyes Del Paso GA. Increased baroreflex sensitivity and reduced cardiovascular reactivity in individuals with chronic low blood pressure. Hypertens Res 2008; 31: 1873-1878.

8 Lohmeier TE, Irwin ED, Rossing MA, Serdar DJ, Kieval RS. Prolonged activation of the baroreflex produces sustained hypotension. Hypertension 2004; 43: 306-311.

9 Fredrikson M, Edman G, Levander SE, Schalling D, Svensson J, Tuomisto M. Electrodermal responsivity in young hypotensive and hypertensive men. Psychophysiology 1990; 27: 649-655.

10 McTavish D, Goa KL. Midodrine. A review of its pharmacological properties and therapeutic use in orthostatic hypotension and secondary hypotensive disorders. Drugs 1989; 38: 757-777.

11 Wright RA, Kaufmann HC, Perera R, Opfer-Gehrking $\mathrm{TL}$, McElligott MA, Sheng KN et al. A double-blind, dose-response study of midodrine in neurogenic orthostatic hypotension. Neurology 1998; 51: 120-124.

12 Duschek S, Heiss H, Buechner B, Werner N, Schandry R, Reyes Del Paso GA. Hemodynamic determinants of chronic hypotension and their modification through vasopressor application. J Physiol Sci 2009; 59: 105-112.

13 Duschek S, Hadjamu M, Schandry R. Enhancement of cerebral blood flow and cognitive performance following pharmacological blood pressure elevation in chronic hypotension. Psychophysiology 2007; 44: 145-153.
14 Duschek S, Heiss H, Werner N, Reyes del Paso GA. Modulations of autonomic cardiovascular control following acute alpha-adrenergic treatment in chronic hypotension. Hypertens Res 2009; 32: 938-943.

15 Bertinieri G, Di Rienzo M, Cavallazzi A, Ferrari AU, Pedotti A, Mancia G. Evaluation of baroreceptor reflex by blood pressure monitoring in unanesthetized cats. Am J Physiol 1988; 254(Pt 2): H377-H383.

16 Parati G, Di Rienzo M, Bertinieri G, Pomidossi G, Casadei R, Groppelli A et al. Evaluation of the baroreceptor-heart rate reflex by 24-hour intra-arterial blood pressure monitoring in humans. Hypertension 1988; 12: 214-222.

17 Parati G, Saul JP, Di Rienzo M, Mancia G. Spectral analysis of blood pressure and heart rate variability in evaluating cardiovascular regulation. A critical appraisal. Hypertension 1995; 25: 1276-1286.

18 Karemaker JM. Last word on point:counterpoint: respiratory sinus arrhythmia is due to a central mechanism vs. respiratory sinus arrhythmia is due to the baroreflex mechanism. J Appl Physiol 2009; 106: 1750.

19 Mancia G, Parati G, Castiglioni P, Di Rienzo M. Effect of sinoaortic denervation on frequency-domain estimates of baroreflex sensitivity in conscious cats. Am J Physiol 1999; 276 (Pt 2): H1987-H1993.

20 Saul JP, Berger RD, Chen MH, Cohen RJ. Transfer function analysis of autonomic regulation. II. Respiratory sinus arrhythmia. Am J Physiol 1989; 256(Pt 2): H153-H161.

21 Julien C. The enigma of Mayer waves: facts and models. Cardiovasc Res 2006; 70: 12-21.

22 Castiglioni P, Di Rienzo M, Veicsteinas A, Parati G, Merati G. Mechanisms of blood pressure and heart rate variability: an insight from low-level paraplegia. $A m \mathrm{~J}$ Physiol Regul Integr Comp Physiol 2007; 292: R1502-R1509.

23 Parati G, Di Rienzo M, Mancia G. How to measure baroreflex sensitivity: from the cardiovascular laboratory to daily life. J Hypertens 2000; 18: 7-19.

24 Parati G, Saul JP, Castiglioni P. Assessing arterial baroreflex control of heart rate: new perspectives. J Hypertens 2004; 22: 1259-1263.

25 Di Rienzo M, Parati G, Castiglioni P, Tordi R, Mancia G, Pedotti A. Baroreflex effectiveness index: an additional measure of baroreflex control of heart rate in daily life. Am J Physiol Regul Integr Comp Physiol 2001; 280: R744-R751.

26 Di Rienzo M, Parati G, Castiglioni P. Assessment of latency in baroreflex control of heart rate during spontaneous behavior. Comput Cardiol 2003; 1: 33-35. 